\title{
Predominio cerebral y rendimiento académico en los estudiantes de la facultad de educación de la Universidad Nacional del Altiplano (UNA)-Puno
}

\section{Brain predomination and academic performance in the students of the education faculty of the National University of Altiplano (UNA) -Puno}

\author{
Myrna Cleofé Sanchez Rossel ${ }^{\text {a }}$ \\ https://orcid.org/0000-0002-0823-2856 \\ Indira Iracema Gómez-Arteta ${ }^{\mathrm{b}}$ \\ https://orcid.org/0000-0001-6489-2261
}

Brisvani Bonifaz Valdez ${ }^{\text {c }}$
https://orcid.org/0000-0002-9067-0731

Escuela Profesional de Educación Secundaria, Universidad Nacional del Altiplano, Puno, Perú.

a msrossel@hotmail.com

b indigoar1@hotmail.com

c diagnosticoedu@gmail.com
Recibido: 16/07/2020

Aceptado: 12/08/2020

\section{Resumen}

El nivel de rendimiento académico y la falta de conciencia sobre el predominio cerebral, dentro del proceso de enseñanza-aprendizaje, fue lo que motivó la realización del presente estudio; cuyo objetivo fue determinar la relación entre el predominio cerebral y el rendimiento académico de los estudiantes de la Facultad de Ciencias de la Educación de la UNA-Puno, durante el semestre 2019-I. Se utilizó el diseño descriptivocorrelacional, enmarcado en el tipo de investigación no experimental y en el enfoque cuantitativo. La muestra (estratificada y proporcional, en base al método aleatorio) estuvo conformada por 305 estudiantes; los que fueron evaluados con los instrumentos: Revelador del Cociente tricerebral, para medir el predominio cerebral y la Ficha de Registro Documental para el rendimiento académico. Al término de la investigación se identificó que el cerebro predomínate es el derecho, con una frecuencia que representa al 52\%. Asimismo, el nivel de rendimiento académico es bueno, con una frecuencia que representa al 49\%. Esto permitió obtener como resultado un valor de $\mathrm{r}=0,321$, que corresponde a una relación moderada entre las variables, confirmándose la hipótesis. Se concluye, así, que el predomino cerebral y el rendimiento académico se asocian moderadamente.

Palabras claves: Predominio cerebral, cociente tricerebral, rendimiento académico y relación.

\begin{abstract}
The he not very optimal level of performance and the lack of awareness about the predominance of the brain, within the teaching-learning process, was what motivated this study; The objective of which was to determine the relationship between brain dominance and academic performance of students from the UNAPuno Faculty of Education Sciences, during the 2019I semester. The descriptive-correlational design was used, framed in the non-experimental type of research and in the quantitative approach. The sample (stratified and proportional, based on the random method) was made up of 305 students; those that were evaluated with the instruments: Developer of the tricerebral quotient, to measure the cerebral predominance and the Documentary Record Card for academic performance. At the end of the investigation, it was identified that the predominant brain is the right, with a frequency that represents $52 \%$. Likewise, the level of academic performance is good, with a frequency that represents $49 \%$. This allowed obtaining as a result a value of $r$ $=0.321$, which corresponds to a moderate relationship between the variables, confirming the hypothesis. Thus, it is concluded that cerebral predominance and academic performance are moderately associated.
\end{abstract}

Keywords: Brain predominance, tricerebral quotient, academic performance and relationship. 


\section{Introducción}

La investigación, centrada en el predominio cerebral y su relación con el rendimiento académico, parte de la premisa de comprender el cerebro, como un gran sistema abierto que abarca otros subsistemas diversos y complejos. (Machado et al., 2013). En ese sentido, el cerebro se convierte en elemento esencial de todo aprendizaje orientado a encaminar acciones investigativas relacionadas con los avances en neurociencias, y así favorecer las habilidades cognitivas (Cantú, Lera y Baca, 2017). Estos avances y los métodos de indagación cerebral, permiten conocer mejor cómo aprende el cerebro, para desarrollar su máximo potencial, $\mathrm{y}$ por ende un mejor rendimiento académico. (Lázaro y Mateos, 2018).

Estudios anteriores han demostrado que los hemisferios cerebrales poseían estructuras y funciones diferentes: el derecho, visual y sintético; el izquierdo, analítico y con buena capacidad de cálculo (Arias, 1999). Además, el hemisferio izquierdo se ocupa del pensar lógico, abstracto y lineal; y el hemisferio derecho, se focaliza en la intuición, la imaginación y lo visual. A la especialización de uno de estos hemisferios en el control de las funciones del individuo se le llama dominancia cerebral (Hernández, 2018). Sin embargo, en muchas ocasiones, el trabajo científico ha priorizado al hemisferio izquierdo, desconociendo que somos seres integrales: creativos, afectivos e instintivos, cualidades del cerebro triuno, dentro de la perspectiva de la evolución tricerebral. (Pérez-Carrero y RodríguezMoreno, 2015). En ese sentido, también Sperry (1981) en su trabajo se refería sólo a los dos hemisferios de la neocorteza; dejando las importantes estructuras cerebrales ubicadas debajo de ella.

Tarantino-Curseri (2018) indica que aunque no se tiene certidumbre del real funcionamiento cerebral, se cuenta con el modelo del «cerebro triuno» de Mac Lean. De acuerdo a él, se tiene tres subcerebros, que evidencian características y funciones distintas y contradictorias entre sí (Borbón, Flórez y Plazas, 2018). Para abrir caminos hacia lo que ha sido llamado «el inconsciente», las investigaciones sobre el cerebro triuno han sido fundamentales, al establecer un orden evolutivo del cerebro en todos los mamíferos superiores: cerebro reptil, paleo mamífero y neo mamífero (Velásquez, Cleves y Calle, 2007). Cabe resaltar que su teoría nunca afirmó que una parte del cerebro actúa independientemente de la otra, solamente es una forma de estructurar el cerebro para poder comprender su funcionamiento, respecto de su desarrollo evolutivo y la complejidad de sus funciones (Quispe, 2015). Siguiendo la línea, la concepción neuropsicológica de Luria (1973-1980) indica que el funcionamiento cerebral se da con la participación integrada de los tres bloques básicos del cerebro, basado en una organización jerárquica de áreas primarias, secundarias y terciarias (Manga y Ramos, 2011). Para Luria, cada zona del cerebro tiene tres funciones predominantes, las que complementadas con las otras partes forman una malla; presentándose la dominancia cerebral, llamada dominancia cíclica; supeditada a la actividad que se esté realizando (Arias, Quintero y Sandoval, 2009). En ese entender, cada sistema es independiente, interactivo e interdependiente; uno de ellos puede ser el dominante, pero los otros dos siempre están involucrados. La independencia de cada estructura cerebral, no implica la negación de las interconexiones obvias; lo que sentimos afecta a lo que pensamos o hacemos y viceversa, conduciéndonos a realizar adecuadamente o no nuestra labor académica (Díaz y Aura, 2009).

Para identificar cuantitativamente el cociente intelectual, emocional y pragmático (cerebro izquierdo, derecho y central) se cuenta con el Revelador del Cociente Tricerebral (CT), instrumento propuesto por De Gregori en 1933. Según los resultados del instrumento, se le designa como cerebro dominante u oficial, al que evidencia el mayor puntaje; al que le sigue en puntaje, se le llama subdominante o antioficial; y al tercero en puntaje, cerebro de apoyo u oscilante. Cuando se tiene el cerebro central dominante se es práctico, organizado, con éxito tanto en el trabajo como en los negocios. Si el dominante es el cerebro derecho, la persona es afectiva, sensible, creativa y soñadora. Cuando domina el cerebro izquierdo, se es un pensador o intelectual (Arias, Quintero y Sandoval, 2009).

Como se observa, existen diversos puntos de vista para analizar el funcionamiento $y$ dominancia del cerebro. Por muchos años, la mayoría de investigadores que escribieron sobre tal dominancia, consideraron que esta se daba en relación a la preferencia de uno u otro de los dos hemisferios de la corteza cerebral. Sin embargo, con otros aportes, se concluye que dominancia cerebral es un constructo que involucra no solo 
a los hemisferios neocorticales y a los límbicos; sino también al metencéfalo, con los hemisferios del cerebelo (Salas, Santos y Parra, 2004). Complementando lo indicado, según Sturges (2015) la versión elemental se enfocaría en el cerebro dividido en dos hemisferios; cada uno subdividido en cuatro partes: lóbulo occipital, temporal, parietal y frontal. Debajo de los hemisferios se ubican las estructuras del sistema límbico y luego el cerebelo, en la parte posterior del cerebro.

Así, la teoría de cerebro tríadico respalda los fundamentos de su desarrollo a través de la evolución del ser humano, distinguiendo la funcionalidad de cada una de sus partes hacia un cerebro integrado, como añadidura a la teoría de dominancia cerebral (González, Peralta, Serrano y Peralta, 2018). Esta propuesta aporta significativamente en la construcción de una nueva pedagogía, que se oriente a potenciar todo el cerebro, para tratar de erradicar prácticas docentes inadecuadas, que atentan contra el desarrollo de la creatividad, por el cultivo, casi exclusivo, del hemisferio izquierdo. A ello se suma, la evaluación escolar inhibidora, que restringe potencialidades, iniciativas e impulsos del estudiante, junto a la imposición de una normativa metodológica tradicional (Torres y Lajo, 2009). De este modo, los docentes se han desenvuelto sabiendo poco o casi nada sobre cómo se produce el aprendizaje en el cerebro de sus educandos; ahora, con el avance de la tecnología, neuropedagogía y la neurofisiología, es viable profundizar en el estudio cerebral y su relación con el proceso de aprendizaje y sus resultados, visibilizados en el rendimiento académico (Quispe, 2015). De allí, la necesidad de comprender el funcionamiento del cerebro y construir un sistema educativo que lo englobe en su totalidad (Ayay, 2017).

En lo que concierne al rendimiento académico, que se mide por medio de calificaciones, en base al sistema de cada universidad (Mamani, 2017), se puede observar como este engloba más de una variable, tanto cognitiva como de personalidad; influida al mismo tiempo por componentes individuales, educativos y familiares. Dichas variables vendrían a constituir las habilidades, conocimientos, actitudes y valores, perfeccionados por el estudiante en el proceso de enseñanza aprendizaje (Fajardo, Maestre, Felipe, León y Polo, 2017). Lo que tiene que ver, según el Ministerio de Educación de Perú, con las capacidades: conocimientos, actitudes y destrezas, como recursos para que los estudiantes puedan desenvolverse de forma competente, ante una situación retadora (Casa, Huatta y Mancha, 2019). Los logros se medirán por medio de calificaciones, con una evaluación cuantitativa, donde se determinan las áreas aprobadas o desaprobadas, en base a los conocimientos, habilidades y actitudes. (Mendives, 2018). Sin embargo, los sistemas nacionales e internacionales de evaluación para medir el rendimiento académico se han centrado solo en los conocimientos, dejando de lado los factores asociados al logro de los estudiantes, ya que dentro del proceso de enseñanza-aprendizaje se articulan múltiples factores que inciden en dicho rendimiento (Cruz, 2016). Actualmente, este tradicional enfoque da un giro; considerando al equilibrio personal como elemento clave para alcanzar un mejor desempeño. Por tanto, conocer las propias emociones y saber ajustarlas ante diversos problemas, será muy importante (Ferragut y Fierro, 2012).

En cuanto a la relación entre predominio cerebral y rendimiento académico, resultados anteriores muestran que muchas personas tienen un hemisferio preferido y esta preferencia afecta la personalidad, las habilidades, y el estilo de aprendizaje, lo que conlleva al rendimiento académico (Rojas, Salas y Jiménez, 2006). Asimismo, indagando los factores etiológicos de las dificultades de aprendizaje, se observa que la causa recae en la falta de dominancia cerebral. Los niños que no logran establecer tal dominancia cerebral presentan problemas en el aprendizaje; por lo que existe una relación significativa entre el rendimiento académico y la dominancia cerebral (Repila, 2014). Y es que la lateralidad incide en el rendimiento escolar, lo que imprimirá el contraste entre los niños con lateralidad definida, más efectivos en sus tareas, a diferencia de aquellos que no cuentan con dicha lateralidad (Rodriguez, 2018). Del mismo modo, el 12.5\% de residentes de medicina tuvieron predominancia del Hemisferio Derecho (HD); 14.6\%, integración hemisférica; $y$ el $35.4 \%$ predominancia del Hemisferio Izquierdo (HI); obteniendo los primeros una calificación promedio significativamente mayor ( $\mathrm{p}=0.03$ ) que los demás (Arias, 1999). Así, el entender que la dominancia cerebral de una persona, hace posible comprender su actuar diferenciador, conlleva a establecer la relación entre dominancia cerebral y logro académico, incidiendo en que la obtención de resultados excelentes, se daría en la medida del reconocimiento de las funciones 
cerebrales, para la optimización del talento humano de cualquier organización (González, Peralta, Serrano y Peralta, 2018). En ese entender, se debe tomar en cuenta la predominancia cerebral, como un aspecto urgente de indagar en la labor docente, ya que esto permitirá llevar a cabo un trabajo cooperativo, para un intercambio fructífero de información e ideas de intervención en el terreno de la lateralidad (Alarcón, 2017). Todo educador debe conocer el funcionamiento del cerebro, para propiciar un equilibrio entre sus diferentes estructuras cerebrales y su desenvolvimiento educativo (Merino, 2016). Pues, se tiene la certeza, ahora, que el cerebro procesa la información que llega a nuestros sentidos y en base a él se establece nuestra conducta social, ética y profesional o académica. Así, cuanto mejor se le conozca, se perfeccionará la interpretación de la realidad; así como la interacción con el contexto ante los retos del siglo XXI (Tarantino-Curseri, 2018). No se debe olvidar que el crecer, aprender, socializar y madurar se relaciona con el ejercicio cerebral, fundamentalmente, con el neocórtex (Arboccó, 2016).

Por lo mencionado anteriormente, el objetivo que guió la investigación fue determinar la relación existente entre el predominio cerebral y el rendimiento académico de los estudiantes de la Facultad de Ciencias de la Educación de la UNA Puno en el semestre académico 2019-I.

\section{Materiales y métodos}

La investigación se realizó en la capital de la región de Puno que se encuentra en el altiplano peruano a $3821 \mathrm{msnm}$, a orillas del lago Titicaca.

La población de estudio enmarcó a 1282 estudiantes de la Facultad de Ciencias de la Educación de la Universidad Nacional del Altiplano-Puno.

Se consideró el muestreo Estratificado y Proporcional, en base al método aleatorio, pues se trabajó con 305 estudiantes de las carreras de Educación Secundaria (73 estudiantes de la especialidad de Lengua, Literatura, Psicología y Filosofía y 49, de Matemática e Informática), Educación Primaria (69 estudiantes), Educación Inicial (62 estudiantes) y Educación Física (52 estudiantes).

Las técnicas utilizadas para la investigación fueron:
- Técnicas de análisis documental, se realizó la revisión de las actas de evaluación de los estudiantes y la base de datos de los docentes para conocer las características específicas y recoger datos de las variables en estudio.

- Técnica de la encuesta, se utilizó para recoger datos, opiniones y percepciones en torno a las variables en estudio para el análisis descriptivo correspondiente.

Los instrumentos utilizados fueron: el Test Revelador del Cociente Mental Tríadico (Rcmt) y la ficha de registro documental, los que se describen a continuación:

\section{Instrumento 1}

El Test Revelador del Cociente Mental Tríadico, cuyo autor es Waldemar De Gregori, es un cuestionario que evalúa el predominio cerebral de los estudiantes y está conformado por 27 preguntas; cada una con una posible puntuación entre 1 y 5, donde cada puntuación debe ser colocada dentro del cuadrado, el rectángulo o el círculo. El procedimiento consiste en sumar los resultados correspondientes a cada uno de los lados; el que obtenga mayor puntaje será el dominante, el inmediatamente inferior será el subdominante, la escala de medición se clasifica como inferior ( 9 a 27 puntos); media (28 a 34 puntos); superior (35 a 39 puntos) y genial 40 a 45 puntos). El test, además tiene en cuenta la ley de la proporcionalidad lo que quiere decir que los lados con menos de dos puntos de diferencia se anulan; mientras que aquellos con diferencia mayor a siete puntos, el más alto domina al más bajo.

\section{Instrumento 2}

La Ficha de Registro Documental o acta de notas es un documento oficial, establecido por la Oficina de Registro Académico de la FCEDUC de la UNAPUNO en el cual se consignan los promedios de las diferentes asignaturas; en base a las capacidades y actitudes que evidencian los estudiantes a lo largo del semestre académico.

Con respecto a la validez y confiabilidad de los instrumentos indicados, el Revelador del cociente tricerebral (CT) de Waldemar De Gregori se validó mediante el estudio titulado "Validación del CTRevelador del Cuociente Tricerebral Nivel 1 (2007) realizado por los investigadores Stella Betancourt, Javier Alberto Castillo Leal y Víctor Julio Pedrozo 
Avilés, cuya conclusión fue que el CT1 puede ser considerado una escala psicosomática válida, susceptible de ser mejorada. Los hallazgos son compatibles con los presupuestos teóricos que derivaron de la construcción de la prueba. Se concluyó también que el Alfa de Crombach, cuyo rango se ubica por encima de 0.81, indica que el instrumento es suficientemente confiable y que puede ser utilizado en los campos de la psicología y la educación.

La Ficha de Registro Documental o acta de notas cumple con la validez de contenido y de constructo por su correspondencia con la teoría utilizada, a través de la evaluación de tres expertos de la Universidad Nacional del Altiplano de Puno, tanto en la forma como en el fondo. A su vez, es un instrumento confiable debido a que los resultados fueron los mismos, en sus repetidas aplicaciones a la muestra de estudio.

Las variables analizadas fueron el predominio cerebral y el rendimiento académico. La primera con el fin de identificar cuantitativamente el predominio del cociente intelectual, emocional o pragmático: cerebro izquierdo, derecho y central, evidenciando tres resultados (uno por cada cerebro). Considerándose, estos, como dimensiones de la variable en mención. La segunda, con el propósito de identificar los calificativos que obtienen los estudiantes en la universidad; ponderados en un sistema vigesimal y que incluyen capacidades y actitudes, que se obtienen mediante exámenes teóricos y prácticos, prácticas, trabajos, y otros calificativos, con la nota de 0 a 20 puntos en lo que respecta a capacidades y el calificativo de 0 a 2 puntos, correspondiente a las actitudes. El promedio se obtiene con la siguiente fórmula: 0,9 (capacidades) + actitudes.

Para cumplir con el objetivo general se trabajó con la prueba del coeficiente de correlación de Pearson y luego se sometió ese resultado a prueba de hipótesis. Todos los cálculos se realizaron con el programa SPSS V.21.

La magnitud de la relación entre las variables se categorizó en:

- $\mathrm{r} \leq 0,25$ Correlación baja

- $r €[0,26-0,50]$ Correlación moderada.

- $\mathrm{r} €[0,51-0,75]$ Correlación alta.

- $r €[0,76-1,00]$ Correlación perfecta o casi perfecta; será perfecta si r es igual a 1 .
- si $r$ es igual a 1. MEJORAMIENTO DE LAS ECUACIONES

\section{Resultados y discusión}

Tabla1.

Predominio cerebral de los estudiantes.

\begin{tabular}{lcc}
\hline $\begin{array}{c}\text { Predominio } \\
\text { Cerebral }\end{array}$ & $\begin{array}{c}\text { frecuencia } \\
(\mathbf{f})\end{array}$ & $\begin{array}{c}\text { porcentaje } \\
(\mathbf{\%})\end{array}$ \\
\hline Izquierdo & 41 & 13 \\
\hline Central & 106 & 35 \\
\hline Derecho & 158 & 52 \\
\hline TOTAL & $\mathbf{3 0 5}$ & $\mathbf{1 0 0}$ \\
\hline
\end{tabular}

Fuente: Revelador del Cociente Tricerebral (CT)

En la tabla 1 se observa que 158 estudiantes que representa el 52\% evidencian un predominio del cerebro derecho. Asimismo, 106 estudiantes que representa al 35\%, evidencian un predominio del cerebro central. Dentro de este grupo, 41 estudiantes, que representa el 13\%, presenta predominio del cerebro izquierdo. Estos resultados se ratifican con los puntajes obtenidos en cada una de las dimensiones consideradas para evaluar el predominio cerebral de los estudiantes, los mismos que se muestran a continuación:

Tabla 2.

Dimensiones para determinar el predominio cerebral de los estudiantes.

\begin{tabular}{llcl}
\hline CEREBRO & DIMENSIONES & $\begin{array}{l}\text { Puntaje } \\
\text { (Promedio) }\end{array}$ & Total \\
\hline Izquierdo & Verbal-numérico & 9 & \multirow{2}{*}{29} \\
\cline { 2 - 3 } & Analítico & 10 & \\
\cline { 2 - 3 } & Crítico-Racional & 11 & \\
\hline Central & Institntivo-práctico & 11 & \\
\cline { 2 - 3 } & Planificador & 12 & \multirow{2}{*}{$\mathbf{3 8}$} \\
\cline { 2 - 3 } & Administrador - & 12 & \\
\hline Derecho & Trabajador & 13 & 12 \\
\cline { 2 - 3 } & Creativo & 13 & \\
\cline { 2 - 3 } & Emocitivo & & \\
\hline
\end{tabular}

Fuente: Revelador del Cociente Tricerebral (CT)

Así el cerebro derecho, vendría a ser el cerebro dominante (oficial), ya que revela el mayor resultado; el subdominante (antioficial), sería el cerebro central; y el tercero, oscilante o cerebro de apoyo, vendría a constituirlo el cerebro izquierdo. Según la teoría de De Gregori, si el dominante es el cerebro derecho, la persona es afectiva, sensible, creativa y soñadora y si el subdominante del derecho es el central, es sensible y práctica; sueña, pero está con los pies 
en la tierra. El desempeño positivo del cerebro izquierdo se manifiesta en las personas sabias, filósofas, pensadoras y científicas; y su desempeño negativo en el ignorante, analfabeta, imbécil o idiota. (Arias, Quintero, \& Sandoval, 2009). Esta posición concuerda con que, es necesario recordar que cada sistema no solamente es independiente, sino también interactivo e interdependiente. Uno de ellos puede ser el dominante, pero los otros dos siempre están involucrados. Pues lo que sentimos afecta aquello que estamos dispuestos a pensar o a hacer; y definitivamente, lo que hacemos afecta la manera en que nos sentimos y contribuye con nuestros procesos de pensamiento, como seres reflexivos que somos, conllevándonos a realizar adecuadamente o no nuestra labor académica (Poca, 2014). En ese sentido, Beltrán, citado por Silvestre (2014) afirma que los estudiantes podrán lograr la creatividad y autonomía en su aprendizaje $\mathrm{y}$ en su desenvolvimiento profesional, siempre que desde cada asignatura se fomente el desarrollo de los tres tipos de pensamiento esenciales; el analítico (analizar, juzgar, criticar, evaluar, comparar y contrastar); el creativo, para que puedan descubrir, inventar, imaginar, elaborar hipótesis, suponer y; el de tipo práctico, para que puedan usar, aplicar, utilizar y practicar (Poca, 2014). Cabe resaltar que, en el campo educativo, por trabajarse con seres humanos, existe la imperiosa necesidad de desarrollar en los docentes en formación, esa capacidad afectiva, sensible, creativa y soñadora, como base de su formación, ya que muchas veces esto se deja de lado, perjudicando el desarrollo integral del estudiante. Así, en contraposición a lo ya explicado, hasta el año 1981, la inteligencia humana era considerada primordialmente una inteligencia racional $\mathrm{y}$, desde la perspectiva de la investigación sobre el cerebro, esta inteligencia ocurría en el hemisferio izquierdo de la neocorteza. (Velásquez, Cleves, \& Calle, 2007).

Tabla 3.

Rendimiento Académico de los Estudiantes.

\begin{tabular}{llll}
\hline $\begin{array}{l}\text { Nivel de Desempeño } \\
\text { Académico }\end{array}$ & Frecuencia & Porcentaje \\
\cline { 3 - 4 } Deficiente & {$[0-10]$} & (f) & $\mathbf{( \% )}$ \\
\hline Regular & {$[11-13]$} & 58 & $5 \%$ \\
\hline Bueno & {$[14-16]$} & 148 & $19 \%$ \\
\hline Muy bueno & {$[17-20]$} & 84 & $28 \%$ \\
\hline TOTAL & & $\mathbf{3 0 5}$ & $\mathbf{1 0 0 \%}$ \\
\hline
\end{tabular}

Fuente: Ficha de Registro Documental.

En la tabla 3 se observa que el mayor porcentaje de estudiantes $49 \%$ demuestra un buen desempeño; seguido del $28 \%$, con un nivel muy bueno; el $19 \%$, evidencia un nivel regular y solo el 5\%, se ubica en el nivel deficiente. Este resultado, evidenciado en las actas como capacidades y actitudes, con notas de 0 a 20 puntos, concuerda con los resultados de (Sánchez, Gómez, Bonifaz y Lujano, 2017), que demuestran un nivel de rendimiento académico bueno, lo que guarda coherencia con la definición de Mamani (2017), quien explica que el rendimiento académico se mide por medio de calificaciones, en base al sistema de cada universidad. Asimismo, con la posición de Fajardo, Maestre, Felipe, León y Polo (2017) quienes ponen de manifiesto que el rendimiento académico engloba más de una variable, tanto cognitiva como la de personalidad; es decir, habilidades, conocimientos, actitudes y valores, perfeccionados por el estudiante en el proceso de enseñanza aprendizaje. Del mismo modo, guarda coherencia con Muñoz, citado por Ramírez y Fuentes (2013), quien indica que el desempeño o rendimiento académico del estudiante es producto tanto de sus conocimientos y habilidades, como de las creencias que tiene acerca de sí mismo. Concuerda también con Mendives (2018), al manifestar que estos logros se medirán por medio de calificaciones, con una evaluación cuantitativa, donde se determinan las áreas aprobadas o desaprobadas, en base a los conocimientos, habilidades y actitudes. Sin embargo, se contrapone a lo siguiente: los sistemas nacionales e internacionales de evaluación para medir el Rendimiento Académico (RA), se han centrado solo en la medición de conocimientos, dejando de lado los factores asociados al logro de los estudiantes (Cruz, 2016). Lo que debe ser superado, de acuerdo a lo fundamentado por varios autores. 
Tabla 4.

Correlación predominio cerebral y rendimiento académico.

\begin{tabular}{llcc}
\hline \multicolumn{4}{c}{ Correlaciones } \\
\hline & \multicolumn{1}{c}{$\begin{array}{c}\text { Predominio } \\
\text { Cerebral }\end{array}$} & $\begin{array}{c}\text { Rendimiento } \\
\text { Académico }\end{array}$ \\
\hline \multirow{2}{*}{$\begin{array}{l}\text { Predominio } \\
\text { Cerebral }\end{array}$} & $\begin{array}{l}\text { Correlación } \\
\text { de Pearson }\end{array}$ & 1 & $0,321^{*}$ \\
\cline { 2 - 4 } & Sig. (bilateral) & 0,047 \\
\cline { 2 - 4 } N & $\begin{array}{l}\text { Correlación } \\
\text { Rendimiento } \\
\text { Académico }\end{array}$ & $0,321^{*}$ & 105 \\
\cline { 2 - 4 } & Sig. (bilateral) & 0,047 & 1 \\
\cline { 2 - 4 } *. La correlación es significativa en el nivel 0,05 (bilateral).
\end{tabular}

Se observa que el coeficiente de correlación de Pearson es $\mathrm{r}=0,321$, ubicado en el intervalo $[0,26-0,50]$ que corresponde a una correlación moderada. Esto quiere decir que las variables predominio cerebral y rendimiento académico de los estudiantes están asociadas o relacionadas moderadamente. Sin embargo, aun cuando la correlación es moderada, es significativa a un $95 \%$ (Significativa al nivel 0,05 ), ya que se tiene una significancia de 0.047 , menor a 0.05 . Esto significa que la correlación encontrada en la muestra se puede aplicar a la población.

Este resultado concuerda con Repila (2014), quien encuentra una relación significativa entre el rendimiento académico y la dominancia cerebral, al explicar que los niños que no logran establecer tal dominancia cerebral presentaban problemas en el aprendizaje. Asimismo, con Rodriguez (2018), cuando indica que la lateralidad o predominio cerebral incide o se relaciona con el rendimiento escolar desde los procesos de aprendizaje, lo que imprimirá el contraste entre los niños con lateralidad definida, más efectivos en sus tareas, a diferencia de aquellos que no cuentan con dicha lateralidad. Sin embargo, hace una atingencia afirmando que niños con lateralidad mal establecida aprueban; pero, manifiesta al mismo tiempo que debe considerarse que los niños que tienen su lateralidad bien definida presentan ventajas en el ámbito escolar. Del mismo modo es coherente con las ideas de Alarcón (2017), ya que este manifiesta que los educadores debemos de tomar en cuenta la predominancia cerebral, como un aspecto urgente de indagar para nuestra labor docente, ya que son muchos de los aspectos del rendimiento académico en los que influye. Asimismo, se alinea al trabajo de Herman quien evidencia que muchas personas tienen un hemisferio preferido y que esta preferencia afecta la personalidad, las habilidades, y el estilo de aprendizaje, lo que conlleva al rendimiento académico (Hannaford, citado por Rojas, Salas y Jimenez (2006). Aunque Herman solo considere el cerebro izquierdo y el derecho. Sin embargo, en contraposición a nuestros resultados al vincular el constructo dominancia cerebral y el rendimiento de los estudiantes de primer año de Ingeniería Civil Industrial de la Universidad de Valparaíso, promoción 2011, en Álgebra y en Cálculo I, se observa que no existe relación significativa (Prado, Sefair y Pasten, 2012), razón por la cual quedaría abierto el debate e investigación sobre este estudio.

\section{Conclusiones}

- La relación que existe entre el predominio cerebral y el nivel de rendimiento académico de los estudiantes de la Facultad de Ciencias de la Educación de la UNA-PUNO, durante el semestre 2019-I, es moderada, ya que el coeficiente de correlación de Pearson es $r=0,321$, el cual recae en el intervalo [0,26$0,50]$. Sin embargo, aun cuando la correlación es moderada, es significativa a un $95 \%$ (Significativa al nivel 0,05 ).

- El cerebro que predomina en el $52 \%$ de los estudiantes en mención es el derecho, con un puntaje promedio de 38 puntos, ubicado dentro de la escala superior, seguido del cerebro central $(35 \%)$, con un promedio de 34 puntos, dentro de la escala media; y, finalmente, el cerebro izquierdo $(13 \%)$, con 29 puntos, situado en la escala inferior. Esto debido a que en ellos prevalecen las actitudes afectivas, la sensibilidad y la creatividad, seguidas del pragmatismo y apoyados por la inteligencia racional.

- El nivel de rendimiento académico de los estudiantes es bueno, con la mayor frecuencia 
que representa al 49\%; de acuerdo al promedio de las capacidades y actitudes, consignadas en las respectivas actas de evaluación.

\section{Agradecimientos}

A la Universidad Nacional del Altiplano, por facilitar la oportunidad de ejecutar investigaciones que ayuden al desarrollo del conocimiento científico.

\section{Referencias bibliográficas}

Alarcón, C. (2017). La importancia de la lateralidad en el rendimiento escolar en educación primaria. Publicaciones Didácticas, (81), 3-25. Recuperado de https:// publicacionesdidacticas.com/hemeroteca/ pd 081 abr.pdf

Arboccó, M. (2016). Neurociencias, educación y salud mental. Propósitos y representaciones, 4(1), 327-362. http://dx.doi.org/10.20511/ pyr2016.

Arias, A., Quintero, E., \& Sandoval, J. (2009). Relación entre la proporcionalidad cerebral triádica y el rendimiento académico de los estudiantes. [Tesis de Maestría en Educación y Desarrollo Humano]. Universidad Manizales-CIDNE. Recuperado de https:// docplayer.es/56739836-Relacion-entre-laproporcionalidad-cerebral-triadica-y-elrendimiento-academico-de-los-estudiantes. html.

Arias, J. (1999). Predominancia de los hemisferios cerebrales en los residentes de medicina. Revista Medica Herediana, 10(1), 21-26. Recuperado de http://www.scielo.org.pe/ scielo.php? $\mathrm{script}=\mathrm{sci}$ arttext\&pid $=\mathrm{S} 1018$ 130X1999000100004\&lng=es\&nrm=iso $>$

Ayay, G. (2017). Método didáctico Socioneuropsicológico y la estimulación de la integración hemisférica cerebral, en educandos del nivel primario. Innoeduca. International Journal of Technology and Educational Innovation, 3(1), 49-56. http:// dx.doi.org/10.24310/innoeduca.2017.

Borbón, M., Flórez, M., \& Plazas, R. (2018). Grafología forense: llave de ingreso al inconsciente criminal. IUSTA, (49), 47-69. $\quad$ http://doi.org/10.15332/19000448.2018.0049.02.

Cantú, D, Lera, J., \& Baca, J. (2017). Especialización hemisférica y estudios sobre lateralidad. Revista de psicología y ciencias del comportamiento de la Unidad Académica de Ciencias Jurídicas y Sociales, 8(2), 6-50. https://doi.org/10.29365/rpcc.20171229-58.

Casa, M., Huatta, S. y Mancha, E. (2019). Aprendizaje Basado en Problemas como estrategia para el desarrollo de competencias en estudiantes de educación secundaria. Comuni@cción, 10(2), 111-121. https:// dx.doi.org/10.33595/2226-1478.10.2.383

Cruz, M. (2016). Factores que influyen en el rendimiento académico del estudiante. Escenarios: empresa y territorio, 5(5), 93-118. Recuperado de http://repositorio. esumer.edu.co/jspui/bitstream/esumer/363/2/ Esumer factores.pdf

Beauport, E. y Diaz, A. (2009). Las tres caras de la mente. El desarrollo de las inteligencias mentales, emocionales y del comportamiento. Venezuela: Alfa. recuperado de https:// psicolog.org/las-tres-caras-de-la-mente.html

Fajardo, F., Maestre, M., Felipe, E., León, B. \& Polo, M. (2017). Análisis del Rendimiento Académico de los Alumnos de Educación. Educación XX1, 20(1) 209-232. https:// dx.doi.org/10.5944/educXX1.14475

Ferragut, M. \& Fierro, A. (2012). Inteligencia emocional, bienestar personal y rendimiento académico en preadolescentes. Revista Latinoamericana de Psicología, 44(3), 95104. Recuperado de https://www.redalyc.org/ articulo.oa? id=80525022008.

González, G., Peralta, O., Serrano, H. y Peralta, G. (2018). Predominio del "cerebro triádico" de acuerdo a niveles de desempeño estratégico de un grupo de trabajadores colombianos. Revista Cubana de Enfermería, 34 (2),56. Recuperado de http://scielo.sld.cu/ scielo.php?script $=$ sci arttext\&pid $=\mathrm{S} 0864$ 03192018000200005\&ling=es\&tlng=es

Hernández, A. (2018). Dominancia cerebral y factores asociados en estudiantes de estomatología de cuarto año. Medisur, 16 (4), 515. Recuperado de http://scielo.sld.cu/ scielo.php?script $=$ sci arttext\&pid $=$ S1727$\underline{897 X 2018000400005 \& \operatorname{lng}=\text { es\&tlng=es. }}$

Lázaro, C. y Mateos, S. (2018). Neurodidáctica en el aula: transformando la educación. Revista Iberoamericana de Educación, 78 (1), 7-8. https://doi.org/10.35362/rie7813296

Machado, S., Arias, O., Orellana, A.,Lattari, E., Cardoso, A. y Egídio, A. (2013). La especialización hemisférica y la regulación de la conducta motora desde la perspectiva de la neurociencia cognitiva. Salud Mental, 
36 (6), 513-520. Recuperado de https://www. redalyc.org/pdf/582/58229682009.pdf

Mamani, T. (2017). Efecto de la adaptabilidad en el rendimiento académico. Educación Superior, 2 (1), 38-44. Recuperado de http:// www.scielo.org.bo/scielo.php?script $=$ sci arttext\&pid=S2518-82832017000100004\&ln $\mathrm{g}=\mathrm{es} \& \mathrm{t} \operatorname{lng}=\mathrm{es}$.

Manga, D. y Ramos, F. (2011). El legado de Luria y la neuropsicología escolar. Psychology, Society \& Education, 3 (1), 1-13. Recuperado de https://www.researchgate. net/publication/277267964 El legado de Luria y la neuropsicologia escolar

Mendives, M. (2018). Las Inteligencias múltiples y su relación con el rendimiento académico en los estudiantes del segundo grado de secundaria de la Institución Educativa Mariscal Castilla -Colán - 2017. Piura. Recuperado de http://repositorio.ucv.edu. pe/bitstream/handle/20.500.12692/28879/ Mendives AMF.pdf? sequence $=1$

Merino, I. (2016). Una nueva vacuna: la vacuna del autoconocimiento. Bases neurobiológicas de la conducta humana. El juego entre el cerebro instintivo-emocional y el cerebro racional. Rev Pediatr Aten Primaria, 18 (70), 85-91. Recuperado de http://scielo.isciii.es/ scielo.php? script $=$ sci arttext\&pid $=$ S113976322016000200017\&奋g=es\&tlng=es.

Muñoz, E. y Gómez, J. (2005). Enfoques de Aprendizaje y Rendimiento Académico de los Estudiantes Universitarios. Revista de Investigación Educativa, 23 (2), 417-432. Recuperado de http://www.redalyc.org/ articulo.oa? id $=283321973007$

Pérez-Carrero, C. y Rodríguez-Moreno, S. y Sánchez-Mayorga, L. (2015). El cerebro tríadico y su relación con la curiosidad, el trabajo en equipoyla explicación de fenómenos para el desarrollo de actitud científica. Rastros y Rostros, 17(31), 99-110. doi: http://dx.doi. org/10.16925/ra.v17i31.1106

Poca, N. (2014). Neurociencias para el aprendizaje en la educación superior. Revista de Investigación Scientia, 3 (1), 10-19. Recuperado de http://50.28.16.6/ investigacion/pdf/4.2.pdf

Prado, C., Sefair, E. y Pasten, V. (2012). Relaciones entre dominancia cerebral, establecimiento educacional de origen $y$ rendimiento académico en matemáticas, en estudiantes de primer año de Ingeniería Civil Industrial de la Universidad de Valparaíso,
Chile. Recuperado de http://www.ifees.net/ wp-content/uploads/2017/07/471.pdf

Quispe, P. (2015). Relación entre Estilos cerebrales de Pensamiento y Aprendizaje autónomo en Estudiantes de Enfermería de la Universidad de Ciencias y Humanidades. Lima. [Tesis de Maestría]. Universidad Nacional de Educación Enrique Guzmán y Valle, Lima, Perú. Recuperada de http:// repositorio.une.edu.pe/bitstream/handle/ UNE/1066/TM\%20CE-Du\%20Q787\%20 2015.pdf? sequence $=1 \&$ is Allowed $=y$

Ramírez, P. y Fuentes, C. (2013). Felicidad y Rendimiento Académico: Efecto Moderador de la Felicidad sobre Indicadores de Selección y Rendimiento Académico de Alumnos de Ingeniería Comercial. Formación Universitaria, 6(3), 21-30 https://dx.doi. org/10.4067/S0718-50062013000300004

Repila, A. (2014). Lateralidad y rendimiento académico, su relación. [Tesis de Maestría] Universidad Internacional de La Rioja, Buenos Aires, Argentina. Recuperada de https://reunir.unir.net/bitstream/handle/ $123456789 / 2335 / 25243$ 15421550552001441655407 TFMAnaRepilaLateralidadyrendimiento. pdf? sequence $=1 \&$ is Allowed $=\mathrm{y}$

Rodriguez, M. (2018). La importancia de la lateralidad en el rendimiento escolar. Revista Ventana Abierta. Recuperado de http://revistaventanaabierta.es/importancialateralidad-rendimiento-escolar/

Rojas, G., Salas, R. y Jiménez, C. (2006). ESTILOS DE APRENDIZAJE Y ESTILOS DE PENSAMIENTO. Estudios Pedagógicos, 3(1), 52. https://doi.org/10.4067/S071807052006000100004.

Salas, R., Santos, A. y Parra, S. (2004). Enfoques de aprendizaje y dominancias cerebrales entre estudiantes universitarios. Aula abierta, (84), 3-22. Recuperado de https://www. researchgate.net/publication/39220515 Enfoques de aprendizaje y dominancias cerebrales entre estudiantes universitarios.

Sanchez, M., Gómez, I., Bonifaz, B., y Lujano, Y. (2017). Concepción del aprendizaje y rendimiento académico en los estudiantes de la Faculta de Ciencias de la Educación de la Universidad Nacional del AltiplanoPuno. Revista de Investigaciones, 6(2), 144-152. Recuperado de http://www. revistaepgunapuno.org/index.php/ investigaciones/issue/view/7/showToc. 
Sturges, P. (2015). Avances de la neurociencia y sus implicaciones para la ciencia de la información. El profesional de la información, 24 (2), 168-175. https://dx.doi.org/10.3145/ epi.2015.mar.10.

Tarantino-Curseri, S. (2018). Pinceladas teóricas en torno al Cerebro Triuno para mejorar nuestra hermenéutica en el ámbito de toda «Negociación». Revista Venezolana de Gerencia, 23 (84), 1-14.

Torres, M. y Lajo, R. (2009). Dominancia Cerebral Asociada al Desempeño Laboral de los
Docentes de una UGEL de Lima. Revista de Investigación en Psicología, 12 (1),84-85. https://doi.org/10.15381/rinvp.v12i1.3782.

Velásquez, B., Cleves, N. y Calle, M. (2007). Determinación del perfil de dominancia cerebral o formas de pensamiento de los estudiantes de primer semestre del programa de bacteriología y laboratorio clínico de la Universidad Colegio mayor de Cundinamarca. Nova, 5 (007), 48-56. 